\title{
Essai de synthèse des recherches sur la cavitation
}

\section{A summary of research work on cavitation}

\author{
G. Gimenez \\ Laboratoire de traitement du signal et ultrasons \\ INSA $502-69621$ Villeurbanne Cedex
}

Contexte général

Essai de définition de la cavitation

D'une manière générale, la cavitation peut être définie comme la formation d'une ou plusieurs poches de gaz (ou "cavités") dans un liquide. C'est par cette définition qu'APFEL commence un article de synthèse récemment publié [1]. Cet auteur souligne également que l'expression "formation de cavité " doit être interprétée soit comme la création d'une nouvelle cavité, soit comme le grossissement d'une cavité déjà existante jusqu'à une taille où des effets macroscopiques peuvent être observés.

Le contenu de la cavité est constitué de vapeur du liquide, de gaz, ou bien d'un mélange de ces deux composants. Les termes "bulle " ou "vide" remplacent souvent celui de "cavité " dans la littérature et on peut dire qu'en français ce sont les mots "bulle " ou "poche" (pour la cavitation développée) qui sont les plus usités.

Une réduction suffisante de la pression du liquide est la cause habituelle de la cavitation mais, évidemment, une élévation de la température au-dessus de la valeur d'ébullition provoque également la formation de cavités. Dans le cas d'une installation donnée, il est important de savoir prédire laquelle de ces deux causes est susceptible d'apparaitre car les dégâts causés par l'une ou l'autre sont de type très différents (érosion et bruit dans la cavitation, fusion de paroi par "burn out " pour l'ébullition) et les rémèdes à apporter pour limiter ces effets néfastes doivent être adaptés en conséquence. Une analyse dimensionnelle de l'ensemble des équations régissant le phénomène de changement de phase permet de caractériser la cavitation, oủ les phénomènes thermiques sont négligeables, de l'ébullition, où les phénomènes thermiques sont prépondé- rants devant les phénomènes dynamiques. Pour être plus précis, la distinction repose sur la valeur de deux groupements adimensionnels qui font intervenir uniquement les propriétés physiques du liquide et de sa phase vapeur (viscosités, densités, chaleurs spécifiques, conductibilités thermiques, chaleur latente et tension superficielle) [2].

\section{Les divers cas de cavitation}

La réduction de pression qui engendre la cavitation se produit principalement dans deux types de situation. Quand la variation de pression qui provoque la cavitation est une conséquence de l'écoulement (stationnaire ou non), on parle de cavitation hydrodynamique (ou hydraulique ou même de cavitation provoquée par un écoulement). Quand la diminution de pression est due au passage d'une onde acoustique dans le liquide on parle de cavitation acoustique ou même de cavitation ultrasonore car la fréquence de l'onde appartient au domaine ultrasonore.

Outre les deux types principaux de cavitation qui viennent d'être mentionnés d'autres apparaissent et se développent graduellement. Il en est ainsi de la cavitation provoquée par la focalisation d'un rayon laser, dénommée cavitation optique. Si les premières expériences datent des années 60 (cf. [3] par exemple), ce sont les nombreux travaux de l'équipe de LAUTERBORN [4] qui ont mieux fait connaitre ce sujet. Les photons utilisés ici pour créer des cavités dans le liquide forment une catégorie particulière de particules. D'autres particules comme les protons ou les neutrinos peuvent également provoquer une

\section{LA HOUILLE BLANCHE/N ${ }^{\circ}$ 5-1984}


rupture du liquide. C'est d'ailleurs ce phénomène qui est utilisé pour détecter de telles particules dans les chambres à bulles. Cette analyse a conduit LAUTERBORN à conclure que la cavitation peut être provoquée par deux mécanismes, soit la tension du liquide (au sens de la tension d'un fil) soit par un apport local d'énergie. Il propose alors la classification schématisée sur la figure 1 .

\section{Les germes de cavitation}

Au début des recherches sur la cavitation, on pensait que ce phénomène correspondait à une rupture, à une déchirure du liquide, de la même manière qu'une éprouvette métallique se rompt lorsqu'on atteint la limite de rupture. L'ordre de grandeur des valeurs des contraintes de traction, improprement mais couramment dénommées " pressions négatives ", nécessaires pour atteindre une telle rupture est facilement évaluable. L'analyse de SIROTYUK [5] est reprise ci-après.

La distance moyenne entre les molécules d'un liquide dépend de la pression et de la température. Dans le cas de l'eau, cette distance est de l'ordre de l'Angström. Quand cette distance moyenne excède une certaine valeur critique, il y a rupture. La théorie cinétique des liquides montre que cette valeur critique est de l'ordre de deux fois la valeur habituelle. En conséquence on peut estimer la valeur de la contrainte de traction correspondant à la limite de rupture à $2 \sigma / R$ où $\sigma$ est la tension superficielle qui représente l'énergie de la surface. Pour de l'eau, avec $\sigma=7,5 \times 10^{-2} \mathrm{~N} / \mathrm{m}$ et $\mathrm{R}=2 \times 10^{-10} \mathrm{~m}$, on trouve une valeur de l'ordre de $10^{4} \mathrm{~atm}$.

Bien évidemment, l'approche précédente est très grossière. En particulier la résistance d'un liquide est abaissée du fait des fluctuations thermiques qui peuvent amener la création spontanée de bulles de vapeur. La prise en compte de ce phénomène (cf. [6]) conduit à estimer à $10^{3}$ atmosphères la valeur pour laquelle se produit la rupture du liquide. Or, dans les études destinées à éprouver la résistance à la rupture de l'eau, les valeurs expérimentales se situent entre 100 atmosphères et 1 atmosphère. Cet important désaccord entre les valeurs théoriques et les valeurs expérimentales a conduit à conclure à l'existence de points faibles dans les liquides, encore appelés germes de cavitation ou nuclei.

Les germes de cavitations sont principalement constitués par des poches de gaz/vapeur "piégées" dans les anfractuosités de particules solides en suspension dans le liquide. On sait qu'il existe aussi des bulles "libres" contenant un mélange de gaz et de vapeur et qui servent évidemment de germes. A ce sujet, il faut indiquer que la raison de la persistance de telles bulles au sein d'un liquide commence seulement à être entrevue (cf. [7]). En effet, si nous considérons une bulle de rayon $R$ en équilibre à un instant donné avec le liquide au repos qui la contient, nous pouvons écrire que la pression $p_{i}$ à l'intérieur de la bulle obéit à :

$$
p_{i}=p+2 \sigma / \rho
$$

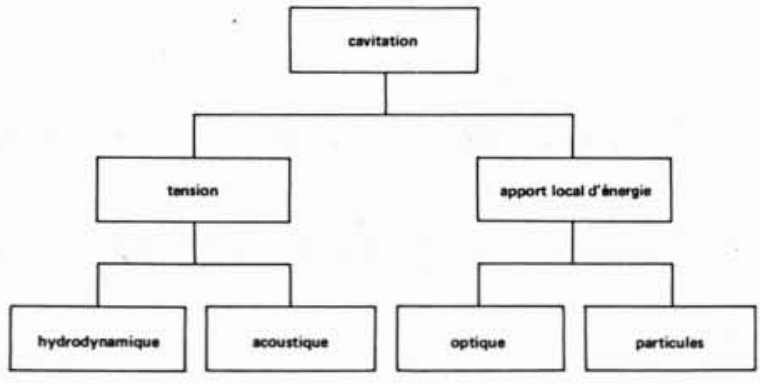

Figure 1 - Classification des divers types de cavitation (après LAUTERBORN [4])

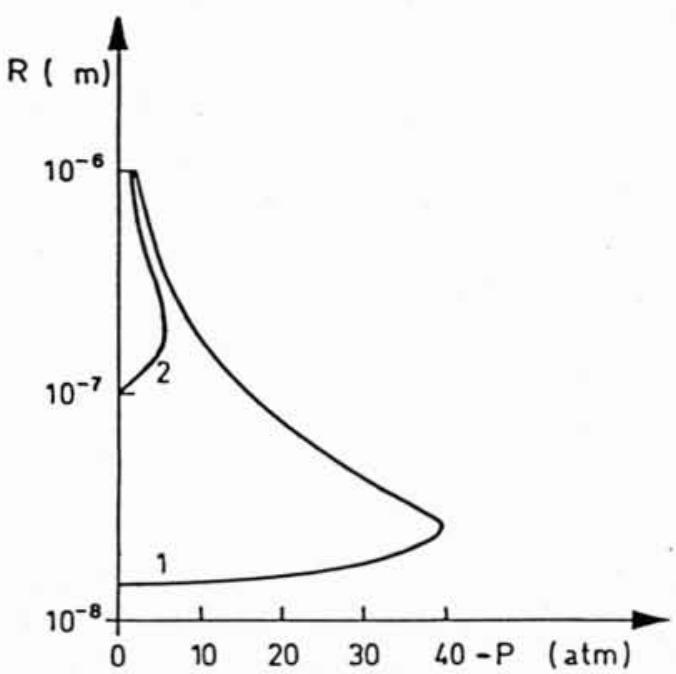

Figure 2 - Variation du rayon d'un germe en fonction de la pression appliquée.

Rayon initial de la bulle: $1,5 \times 10^{-8} \mathrm{~m}$ (courbe 1 ) et $10^{-7} \mathrm{~m}$ (courbe 2).

(après SIROTYUK [5])

En conséquence la pression à l'intérieur de la bulle excède la pression $p$ dans le liquide et le gaz devrait diffuser de la bulle vers le liquide entrainant la disparition de celle-ci ( $c f .[8])$. Ce phénomène devrait être d'autant plus rapide que le rayon de la bulle est faible. Or certains travaux montrent qu'un liquide au repos contient du gaz sous forme libre (bulles) même après plusieurs jours, voire plusieurs semaines de repos. Cela est vraisemblablement dû au blocage de la diffusion gazeuse par la présence de traces de polymères.

L'attitude des expérimentateurs vis à vis du problème des germes diffère suivant le domaine dans lequel ils travaillent. D'un côté, pour les recherches relatives à la résistance, à la rupture des liquides, on est amené à purifier les liquides et à les conserver purs. On aura une idée de la difficulté de satisfaire cette seconde contrainte quand on saura que la production d'eau absolument pure est impossible. C'est en effet un des solvants les plus puissants qui dissout les parois du récipient qui le contient et les gaz avec lesquels il est amené à être en contact. D'un 
autre côté, pour les expérimentateurs concernés par la cavitation dans des dispositifs industriels, la question des germes est plutôt liée à la reproductibilité des résultats ou aux problèmes de similitude. ils sont alors amenés à déterminer les distributions de germes par des méthodes acoustiques (cf. [9]), optiques (cf. [10]), électriques (cf. [11] et [12]) ou même en mesurant directement la pression critique des germes au moyen d'un Venturi ou d'une veine étalon (cf. [13]).

\section{Dynamique des bulles}

La majeure partie des effets de la cavitation est liée plus ou moins directement à la dynamique de la bulle, c'est-à-dire à l'évolution de sa taille en fonction du temps (on parle également de mouvement radial dans le cas de bulle sphérique). Dans ce qui suit nous allons montrer, dans le cadre d'une modélisation extrêmement grossière, comment une diminution de pression peut conduire à un grossissement "indéfini " d'une bulle puis à son implosion. Considérons donc une bulle isolée et en équilibre au sein d'un liquide soumis à la pression $p$. Il a déjà été indiqué que la pression $p_{i}$ à l'intérieur de la bulle vérifie l'égalité (1). La bulle contenant du gaz et de la vapeur, la pression $p_{i}$ sera donnée par :

$$
p_{i}=p_{k}+p_{i v}
$$

où $p_{g}$ et $p_{v}$ sont les pressions partielles respectives du gaz et de la vapeur. Pour une telle bulle, en considérant que la pression $p_{i}$ est donnée par l'égalité (1) et en utilisant la valeur de $p_{r}$ à $20^{\circ} \mathrm{C}\left(2,3 \times 10^{3} \mathrm{~Pa}\right)$ on peut montrer que le rapport du nombre des molécules de gaz à celui des molécules de vapeur est au minimum de l'ordre de 50 . On peut donc considérer que l'influence de la vapeur peut être négligée.

Dans ce qui suit nous allons ignorer la dynamique du problème en imaginant une expérience quasi-statique durant laquelle la pression du liquide diminuerait d'une manière suffisamment lente pour que la variation de rayon de la bulle s'effectue également très lentement $(\mathrm{dR} / \mathrm{dt} \simeq 0)$. On peut alors considérer que l'évolution du contenu gazeux de la bulle est isotherme et écrire :

$$
p_{i}=p_{0}\left(\frac{R_{0}}{R}\right)^{3}=\left(p_{0}+\frac{2 \sigma}{R_{0}}\right)\left(\frac{R_{0}}{R}\right)^{3}
$$

Dans cette expression, l'indice 0 indique que la grandeur indicée a sa valeur initiale. Comme $p_{i}$ vérifie la relation (1) :

$$
p=\left(p_{0}+\frac{2 \sigma}{R_{0}}\right)\left(\frac{R_{0}}{R}\right)^{3}-\frac{2 \sigma}{R} .
$$

Dans ces expressions $p_{i c}$ représente donc la valeur de $p_{i}$ à l'instant initial, instant où on commence à diminuer la pression $p$ du liquide depuis sa valeur initiale $p_{o}$. En fixant la valeur de $p_{o}$ (nous prendrons $10^{5} \mathrm{~Pa}$ ), l'expression (4) permet de calculer la fonction $p(R)$ pour diverses valeurs du rayon initial $R_{i}$. Les courbes $R(p)$ qui en découlent sont présentées sur la figure 2 . Notons que la valeur de $p$ devient presque tout de suite négative. Ces courbes comportent une zone stable en dessous du point où la tangente est verticale et une zone instable au-dessus de ce point. En reprenant le cas d'une diminution lente de la pression à partir de sa valeur initiale, on observerait qu'à la diminution de pression correspondrait une augmentation régulière du rayon de la bulle jusqu'à ce qu'on atteigne le point d'instabilité. A ce moment la bulle se mettrait à grossir "indéfiniment ". Les coordonnées du point d'instabilité $\left(\boldsymbol{p}_{c}, \boldsymbol{R}_{c}\right)$ se déduisent de l'expression (4) en écrivant que

$$
\left(\frac{d p}{d R}\right)_{R=R_{c}}=0
$$

On obtient :

$$
R_{c}=R_{0}\left[\frac{3 R_{0}}{2 \sigma}\left(p_{0}+\frac{2 \sigma}{R_{0}}\right)\right]^{1 / 2},
$$

et

$p_{c}=-2\left[k^{3} /\left(p_{0}+k\right)\right]^{1 / 2}$

avec $k=2 \sigma / R_{0}$

Les résultats précédents peuvent s'énoncer de deux manières. On peut dire que pour un germe donné $\left(R_{0}=\right.$ constante), il faut que la pression soit inférieure à une valeur critique $p_{c}\left(R_{0}\right)$ pour que le germe perde sa stabilité; mais on peut aussi dire qu'une pression donnée $p_{c}$, appliquée à une population de germes ( $R_{0}$ variable), produit le grossissement des seuls germes pour lesquels $R_{0}$ est supérieur à $R_{r}$

Bien entendu, avoir négligé l'inertie a considérablement éloigné le problème traité de la réalité physique. Néanmoins l'existence d'un rayon minimal de germe est suggéré.

Dans les cas réels de cavitation, la valeur critique de la pression est franchie $\left(p<p_{c}\right)$ sur une certaine longueur, donc un certain temps (cas de la cavitation hydrodynamique), ou directement pendant un certain temps (cas de la cavitation ultrasonore). Par ailleurs, lorsque la pression n'excède plus la pression critique, la phase de grossissement est arrêtée et la phase d'implosion commence. Notons qu'à ce moment, le volume de la bulle est si important par rapport à son volume initial que la bulle est quasiment "vide", le mouvement est alors uniquement contrôlé par l'inertie du liquide. La phase d'implosion se termine quand la pression dans la bulle est devenue si élevée, du fait de la compression, que le mouvement du liquide est arrêté. En schématisant, on peut dire que l'implosion est d'autant plus violente que le rayon maximal atteint par la bulle est élevé vis à vis de son rayon initial. On voit alors qu'un paramètre particulièrement important est la durée de la phase de grossissement, c'est-à-dire la durée pendant laquelle la pression est inférieure à la pression critique.

\section{Chronologie des études} de la dynamique des bulles(*)

La présentation d'une chronologie sommaire des études de la dynamique des bulles permet de voir comment des

(*) La rédaction de cette partie doit beaucoup aux indications puisées dans la thèse de CHAHINE [14]. 
modélisations aussi sommaires que celle exposée précédemment ont peu à peu cédé la place à des théories plus élaborées prenant à chaque fois en compte un élément nouveau.

La première approche théorique connue remonte à 1859 et est due à BESANT [15]. RAYLEIGH [16], ayant repris en 1917 cette étude, a vu depuis son nom associé à toute approche théorique de la cavitation. Un intérêt croissant a valu, particulièrement après 1940 , un nombre très important de publications. L'ouvrage de KNAPP, DAILY et HAMMITT (1970) [17], ainsi que l'article de synthèse de PLESSET et PROSPERETTI (1977) [18], ne donnent qu'une idée des travaux connus. ROBERTSON [19] estime en 1969 à plus de dix mille le nombre des publications concernant la cavitation. En se limitant à l'aspect fondamental du phénomène la chronologie suivante peut être indiquée.

RAYLEIGH (1917) [16], étudie le champ de pression crée, dans un fluide parfait, incompressible, au repos, par la disparition d'une cavité sphérique vide, sous l'effet d'une pression hydrostatique constante donnée. Il recherche ensuite l'influence de la présence d'un gaz dans la cavité.

COOK et PARSONS (1919) [20], abandonnent l'hypothèse de l'incompressibilité du liquide vers la fin de l'implosion, mais supposent que le champ de pression produit est celui qui est créé par l'implosion de la bulle sur une sphère solide fictive concentrique et de rayon donné très faible. Cette hypothèse permet le calcul d'une pression caractéristique de l'implosion, appelée par les auteurs " pression marteau ".

Il faut attendre plus de vingt ans pour que ces études soient poursuivies.

HERRING (1941) [21], s'intéressant aux explosions sous-marines, étudie les pulsations de la bulle créée par ces explosions et l'influence sur ces pulsations de la présence du fond, de la surface libre ainsi que l'effet de la gravité.

BEECHING (1942) [22], reprend les équations de RAYLEIGH et y ajoute les termes correspondants à la tension superficielle et à la présence des gaz dans la bulle.

SILVER (1942) [23], effectue de son côté des calculs élaborés tenant compte des effets thermodynamiques associés à l'échauffement dû à la compression de la vapeur et aux transferts de chaleur.

Tous ces modèles, comme on le constate, admettent la symétrie sphérique du problème, imputant implicitement l'érosion à une surpression ou à des ondes de chocs au sein du fluide.

KORNFELD ET SUVOROV (1944) [24], vont cependant les premiers suggérer l'existence d'un jet rentrant durant l'implosion pour expliquer l'érosion de paroi.

COLE (1948) [25], écrit les équations de la dynamique du grossissement des bulles aussi bien dans un fluide incompressible que dans un fluide compressible. Utilisant ensuite la méthode des images, il étudie l'influence des parois solides ou des surfaces libres voisines.

PLESSET (1948) [26], étudie les variations du rayon d'une bulle dans un champ de pression donné, en tenant compte de la viscosité, et établit une équation connue sous le nom d'équation de RAYLEIGH-PLESSET. L'application de sa théorie au cas d'un germe transporté dans un écoulement se fait en considérant que la bulle suit une ligne de courant. Il admet que l'évolution du rayon est directement liée à l'évolution de la pression sur cette ligne de courant, la bulle ne perturbant pas l'écoulement global en première approximation.

KNAPP et HOLLANDER (1948), effectuent la même année le premier travail expérimental important. L'utilisation de la cinématographie ultra-rapide leur permet de mettre en évidence l'existence de vitesses et d'accélération radiales importantes, lors de l'implosion.

NOLTINGK et NEPPIRAS (1950) [28], font les premiers calculs numériques à partir de l'équation de RAYLEIGH-PLESSET [26] dans le cas d'un champ de pression sinusoïdal. Ils montrent alors que le rayon de la bulle passe par un maximum avant que l'implosion ne se produise. Pour certains rayons de bulle, ils constatent que le grossissement et l'implosion se produisent au cours d'un seul cycle acoustique.

TRILLING (1952) [29], étudie l'implosion et le rebondissement d'une bulle contenant un gaz parfait se comprimant adiabatiquement. Il admet que les perturbations de pression se propagent dans le liquide à la vitesse du son et considère une onde de compression à l'intérieur de la bulle et une onde de détente dans le liquide.

GILMORE (1952) [30], ne néglige pas comme TRILLING les vitesses locales du liquide devant la célérité du son et, considérant une compression isentropique, il réécrit les équations de RAYLEIGH en tenant compte de la compressibilité.

NAUDE et ELLIS (1961) [31], développent en harmoniques sphériques l'écart par rapport à la forme sphérique et prévoient la formation d'un jet rentrant. Ils visualisent pour la première fois, à l'aide de la cinématographie ultra-rapide, la formation de ce jet.

Depuis les années 60, l'étude de la cavitation s'accélère avec l'intérêt croissant que commencent à lui porter ceux qui s'intéressent aux ultrasons et aux écoulements diphasiques. De même l'essor de l'informatique va permettre aux chercheurs d'aborder numériquement le problème des déformations de la surface de la bulle au voisinage de parois.

FLYNN (1964) [32], montre qu'une bulle peut entrer en résonance avec le champ de pression sinusoïdal imposé et grossir pendant plusieurs cycles pour imploser ensuite.

FLORSCHUETZ et CHAO (1965) [33], cherchent à déterminer dans quelles conditions on peut négliger les transferts de chaleur pour l'étude de la dynamique des bulles. Ils établissent un critère de prédominance des effets d'inertie sur les effets des transferts thermiques.

PLESSET et CHAPMAN (1969) [34], utilisant une méthode de différences finies, étudient numériquement, dans un fluide parfait, l'implosion d'une bulle au voisinage d'une paroi solide. Ils mettent ainsi en évidence l'apparition et le développement d'un jet rentrant.

MITCHELL et HAMMITT (1970) [35], étudient de leur côté le cas d'un fluide visqueux. Le cas de l'implosion dans un gradient de pression est également considéré, et la naissance du jet est mise en évidence.

AKULICHEV (1971) [36], montre que les équations qui gouvernent le mouvement radial des bulles sphériques, en cavitation ultrasonore, présentent des "zones d'instabilité " : des variations très faibles de certains paramètres produisent des variations très brutales des solutions numériques. 
HSIEH (1972) [37], présente une nouvelle approche théorique de la dynamique des bulles non sphériques. Il écrit une formulation variationnelle du problème ouvrant ainsi la voie à des nouveaux développements. Il considère de plus les effets de transferts de masse et de chaleur ainsi que les effets de compressibilité pour les bulles quasisphériques.

\section{Effets de la cavitation}

Schématiquement les effets de la cavitation peuvent être regroupés en cinq classes :

- effet acoustique : un sifflement caractéristique est émis à partir de la zone de cavitation.

- effet mécanique: les solides immergés dans le liquide sont érodés, des capteurs de pression enregistrent des impulsions de forte amplitude, les écoulements sont modifiés.

- effet lumineux : de la lumière est émise par la zone de cavitation.

- effet chimique : des réactions qui se produisaient avant cavitation s'accélèrent, d'autres qui n'existaient pas se déclenchent.

- effet électrique: des capteurs appropriés décelent des modifications des propriétés électriques du milieu et l'application de champs magnétique ou électrique modifie certains des effets cités plus haut.

Donnons quelques indications supplémentaires au sujet de l'effet mécanique d'érosion car ses conséquences sont importantes dans les dispositifs hydrodynamiques; pour cette raison, c'est sans aucun doute le domaine oú les études et les essais ont été les plus nombreux. Ce sont d'ailleurs les problèmes d'érosion des hélices qui ont conduit LORD RAYLEIGH [16] à présenter la première étude théorique du phénomène.

Si la cavitation hydrodynamique fut la première étudiée, on s'aperçut rapidement que la cavitation ultrasonore produit une érosion plus rapide. Cet avantage expérimental, des matériels moins coûteux et moins encombrants que ceux de la cavitation hydrodynamique, sont à l'origine du développement des équipements de cavitation ultrasonore. Néanmoins, la comparaison des résultats fournis par les deux méthodes a toujours été délicate, ainsi que l'a souligné NUMACHI [38]. Cette question a d'ailleurs fait l'objet, en 1976, d'une partie des discussions d'une rencontre de spécialistes [39].

Si au début des études, l'érosion était évaluée par des méthodes assez empiriques, comme par exemple l'augmentation de transparence d'une fine feuille de métal (DEGROIS et coll. [40], BOUCHER et coll. [41]), à présent les grandeurs mesurées sont au nombre de trois. Le plus souvent c'est la perte de masse de l'échantillon qui est évaluée, mais on trouve également le volume du métal enlevé ou la profondeur moyenne de pénétration (MDPR : mean depth of penetration) qui est le quotient du volume perdu par l'aire exposée à la cavitation.

Malgré des difficultés de comparaison des divers travaux expérimentaux, certains résultats semblent se dégager des études effectuées. La variation de l'érosion en fonction du temps (existence d'une durée d'incubation) est souvent observée. L'existence d'un maximum de l'érosion en fonction de la température a été observée par HAMMITT [42]. Un tel maximum apparaît également dans les mesures de BEBCHUK $[43,44]$ ainsi que dans celles de PLESSET [45]. Dans ce dernier travail, la position du maximum évolue entre $20^{\circ} \mathrm{C}$ et $60^{\circ} \mathrm{C}$ suivant le liquide utilisé (eau distillée, eau tamponnée de $\mathrm{PH} 8$, solution à $3 \%$ de $\mathrm{NaCl}$ ). A notre connaissance, seule l'étude de KORTNEV et coll. [46] inclut la variation du paramètre $\alpha$, teneur en gaz du liquide. L'importance du contenu gazeux est nettement démontrée par ces auteurs.

Un autre résultat, de nature plus technologique, a été le classement (GARCIA et coll. [47], KNAPP et coll [48]) des métaux utilisés dans les dispositifs industriels selon leur résistance à la cavitation.

Si la majorité des chercheurs s'accorde pour reconnaître dans le mouvement des bulles la cause des effets érosifs, des divergences subsistent sur le processus exact d'enlèvement de matière. Une de ces divergences, rapportée par FLYNN, [32] porte sur la position des bulles, celles-ci peuvent se trouver au sein du liquide, ou bien être fixées sur la surface qui s'érode. Dans le premier cas, l'érosion peut être provoquée par l'onde de choc se propageant après l'implosion de la bulle ou par jet de liquide de grande vitesse penant naissance lorsque la bulle perd sa symétrie sphérique, ainsi que l'a montré KORNFELD [24]. Dans le second cas, les dommages peuvent provenir de la forte pression ou de la forte température régnant dans le gaz ou encore de l'impact du liquide sur la surface.

\section{Inconvénients de la cavitation}

Avec le développement des performances, les effets néfastes de la cavitation se manifestent dans des situations industrielles extrêmement variées. Citons quelques exemples à titre d'illustrations :

Génie maritime: Surfaces portantes d'hydroptère, hélices rapides, safran de stabilisation, faces parlantes de sonar. Génie industriel: Cavitation des lubrifiants sous l'action des vibrations d'arbres, pompes, arbres de turbines hydrauliques.

Génie pétrolier: Coudes des conduits de déchargement en transport rapide d'hydrocarbures, clapotis dans les cuves de gaz liquéfié (méthaniers par exemple).

Génie nucléaire : Cavitation du sodium liquide au pied des assemblages dans les cœurs de pile à neutrons rapides, circuits de refroidissement.

Génie civil: Cavitation derrière les diaphragmes, dans les vannes de fermeture de barrages, canalisations.

Détaillons quelques effets néfastes qui perturbent le bon fonctionnement des dispositifs.

1) L'érosion de cavitation: les cavités de vapeur créées dans les zones de forte dépression sont le siège du phénomène de changement de phase inverse vapeur-liquide lorsque ces cavités sont convectées par l'écoulement dans des zones de recompression. Au moment de la recompression, les cavités de vapeur implosent. Il s'ensuit 
des pics de pression intense. De plus, en présence d'une paroi cette implosion s'effectue avec création d'un jet rentrant orienté vers la paroi et animé d'une vitesse pouvant être suffisamment élevée pour créer des dommages importants sur les matériaux.

2) La perte de performances: l'existence d'une cavitation suffisamment développée limite les différences de pression que l'on calcule entre intrados et extrados des profils d'hydroptères ou des pales d'hélice. En adoptant une formulation différente, on peut dire que la cavitation est un facteur limitant essentiel dans la conception des machines hydrauliques.

3) Le bruit de cavitation: l'implosion des poches de vapeur dans les zones de recompression s'accompagne toujours d'un bruit caractéristique intense, qui gêne considérablement les méthodes auditives de diagnostic du fonctionnement des machines (par exemple, l'analyse du bruit d'ébullition comme moyen de détection des accidents de réacteurs nucléaires) et qui diminue sensiblement la discrétion d'utilisation, ce qui peut avoir des conséquences graves au plan de l'armement (hélices de sous-marins par exemple).

\section{Applications de la cavitation}

Dans de nombreux cas, on cherche à provoquer la cavitation. Tout d'abord c'est un moyen de caractériser le milieu liquide. Dans les liquides très soigneusement purifiés le seuil de cavitation peut approcher ou même égaler la valeur de la résistance à la rupture du liquide. Pour les liquides moins purs, l'importance de la cavitation est directement liée aux impuretés contenus dans le liquide, à sa teneur en gaz et à son "histoire ". Par ailleurs, l'apparition " ponctuelle " de cavitation peut être provoquée par des rayonnements ionisants et par là-même constituer une méthode d'étude de ces rayonnements.

Il existe de nombreuses autres applications de la cavitation qui sont pour la plupart mises en œuvre dans des dispositifs de cavitation ultrasonore. Ces applications découlent du fait qu'avec des densités d'énergie relativement faibles, on obtient des implosions de bulles conduisant à des pressions de plusieurs milliers d'atmosphères et à des températures de plusieurs milliers de degrés Kelvin. Autrement dit la cavitation permet une concentration d'énergie dans les milieux liquides.

Parmi les applications de la cavitation on peut citer :

- le dégazage des liquides, qu'il s'agisse du passage du gaz de la forme dissoute à la forme libre (bulles) ou de l'élimination des bulles;

- les processus où la diffusion gazeuse et les transferts thermiques de conduction interviennent car les bulles créées se déplacent dans les champs de pression inhomogènes et détruisent les couches-limite;

- les dépôts par électrolyse dont l'uniformité est améliorée et la vitesse fortement augmentée car les bulles brisent les couches d'ions qui entourent les électrodes; - le nettoyage par ultrasons, l'une des applications les plus largement répandues;
- la dispersion de particules solides dans les liquides (cosmétiques, peintures);

- la production d'émulsion;

- la destruction de cellules vivantes (la membrane est déchirée) qui permet l'extraction du contenu de la cellule ou la destruction de bactéries.

La cavitation hydrodynamique donne également lieu à des applications :

- jets cavitants utilisés pour le forage, le dépotage des mines ou le nettoyage;

- limiteur de débit fonctionnant par effet de blocage lorsque la cavitation apparait;

- source de bruit intense (leurre acoustique).

\section{Situation actuelle des recherches}

Plus d'un demi-siècle après les premières recherches sur la cavitation, trois questions fondamentales restent posées :

- quelles sont les bonnes lois de similitude à respecter entre modèle et réel ?

- comment prévoir puis limiter l'érosion?

- quel modèle théorique explique au mieux les effets de cavitation?

\section{Similitude}

On admet depuis plus de trente ans, que la similitude entre modèle et réel est réalisé si le paramètre de cavitation $\sigma$ $=\left(P_{\infty}-P_{v}\right) /\left(1 / 2 \rho V^{2}\right)$ est conservé. En fait, il s'agit d'une condition nécessaire mais pas suffisante. C'est ainsi que la comparaison des résultats obtenus pour un même profil, mais dans des installations différentes, est loin d'être satisfaisante [49]. Il en est de même des résultats obtenus, dans la même installation, pour des profils semblables de tailles différentes ou de matériaux (donc d'états de surface) différents [36]. Plus significative encore est la différence enre les résultats obtenus, dans une même installation et pour un même profil, mais avec des procédures de mise en route des essais différentes. Les courbes qui donnent $\sigma$ en fonction du nombre de Reynolds présentées par KELLER [51] au "Cavitation and Polyphase Flow Forum 1978 " sont très éloquentes à ce sujet. La comparaison des résultats montre que, pour un même Reynolds, la valeur du paramètre $\sigma$ pour laquelle apparait la cavitation peut être décuplée d'une installation à une autre.

Trois causes qui ne sont pas totalement indépendantes peuvent expliquer ces problèmes d'échelle. La première concerne l'influence de la couche limite mise en évidence par ARAKERI et ACOSTA [52, 53]. La seconde raison de disparité entre les mesures peut résider dans les différents niveaux de turbulence entre les divers tunnels de cavitation utilisés [54]. La troisième raison concerne l'importance des germes sur la similitude de cavitation. En effet, s'il est nécessaire de connaitre la distribution des germes, il faut aussi comprendre au mieux leur comportement dans un champ de pression. 


\section{Prévision de l'érosion de cavitation}

Deux techniques sont actuellement utilisées pour tenter de prévoir quantitativement l'érosion de cavitation. La plus courante consiste à corréler le bruit émis par la zone de cavitation, et l'érosion. Bien qu'il semble qu'une relation quasi-linéaire existe entre le bruit total et le taux d'érosion (perte de masse par unité de temps), l'utilisation de cette technique pose certaines questions. Par exemple, il n'existe pour l'instant aucun moyen de distinguer acoustiquement une bulle "érodante" d'une autre qui ne l'est pas. Ou encore : l'érosion d'un échantillon introduit dans la zone de cavitation dépend fortement de la position exacte de l'échantillon; or, l'analyse du bruit de cavitation, qui prend en compte l'ensemble de la zone de cavitation, ne peut conduire qu'à une sorte de valeur moyenne du taux d'érosion.

Une autre technique, plus récente, consiste à analyser, au moyen de capteurs piezoélectriques miniatures, les impulsions de pression consécutives à l'implosion des bulles. NUMACHI [38], en 1965, s'aperçut le premier que les diverses impulsions de pression présentent des amplitudes différentes. Mais c'est MAKAROV et coll. [55, 56] qui ont obtenu les premiers histogrammes d'amplitude, puis qui ont montré que ces histogrammes sont corrélés aux pertes de masse d'un échantillon métallique substitué au capteur piézoélectrique miniature. Il faut cependant souligner que cette méthode doit encore être étudiée et également rendue plus fiable pour sortir du cadre des essais en laboratoire.

\section{Modélisation de la cavitation}

On peut schématiquement regrouper les questions qui se posent encore à propos du comportement des bulles de cavitation en quatre classes, les trois premières faisant référence à un moment particulier de la "vie " des bulles.

\section{Origine de la bulle de cavitation}

Comme il a été indiqué précédemment, en cavitation naissante, les bulles de cavitation ont pour origine des microbulles (ou germes de cavitation), qui sont déjà présentes dans le liquide au repos. A propos de ces germes, plusieurs points n'ont pas été éclaircis de manière satisfaisante :

- les germes peuvent-ils exister à l'état libre au sein du liquide (microbulles sphériques), ou sont-ils toujours associés à des particules solides (gaz piégé dans les anfractuosités d'une paroi par exemple)?

- par quel mécanisme la taille des germes "libres" est-elle stabilisée dans le liquide au repos (pour les germes " piégés ", le mécanisme est connu) ?
- comment les germes sont-ils distribués (répartition du nombre de germes d'une taille donnée en fonction de cette taille)?

- comment la distribution évolue-t-elle quand le liquide passe de l'état de repos à des conditions expérimentales données?

\section{Dynamique de la bulle de cavitation hors implosion}

Dans ce domaine, beaucoup de travail a été accompli et beaucoup de résultats ont été obtenus. Néanmoins, dans le cas d'une détermination expérimentale du mouvement d'une bulle, il n'a pas été possible d'ajuster les paramètres des équations du mouvement afin que la solution concorde avec le mouvement effectivement observé. Il convient donc de rechercher les causes de cette divergence.

\section{Phase finale de l'implosion}

C'est la phase importante car c'est le mouvement pendant cette phase qui est à l'origine de l'érosion de cavitation. De plus, c'est durant cette période que beaucoup d'équations donnent des résultats physiquement non satisfaisants. Il convient donc de modifier les équations existantes en y intégrant des termes jusque là négligés. Il ne faut cependant pas se cacher que le problème principal est celui de la confrontation entre le modèle et les résultats expérimentaux car cette phase finale de l'implosion est extrêmement brève : au maximum de l'ordre de la nanoseconde. Dans le cas d'une bulle sphérique, on peut définir cette phase finale comme commençant au moment où la vitesse de la paroi de la bulle dépasse la vitesse du son et se terminant quant la vitesse de paroi s'annule, juste avant que la bulle ne grossisse de nouveau.

Une fois réglé le problème précédent, il faudra calculer le champ de pression au voisinage du lieu de l'implosion, aussi bien quand celle-ci se produit au voisinage d'une paroi qu'au sein du liquide. On pourra sans doute alors faire la part, pour ce qui concerne les dommages causés par la cavitation, entre les dégâts causés par les jets de liquide et ceux causés par les ondes de choc.

Il restera ensuite à savoir ce que devient la bulle après l'implosion. Se fractionne-t-elle en plusieurs bulles plus petites, ou reste-t-elle intacte et recommence-t-elle à grossir pour imploser de nouveau?

\section{Passage à un modèle à plusieurs bulles}

Ce qui précède concerne le cas d'une bulle isolée. Or, dans la réalité, il y a toujours un grand nombre de bulles mises en jeu. Certains auteurs, comme CHAHINE [57], ont déjà abordé le sujet. Il est cependant vraisemblable que la prise en compte progressive de l'interaction des bulles progressera notablement dans l'avenir. Presque tout est à faire dans ce domaine. 


\section{Confexte national}

Les recherches sur la cavitation, en France, sont le fait de laboratoires de type universitaire, de grands organismes comme le Commissariat à l'Energie Atomique ou Electricité de France et d'industriels comme, par exemple, la Société NEYRTEC. De plus, des études à vocation militaire sont menées dans des organismes comme le Bassin des Carènes. Enfin, la Direction des Recherches Etudes et Techniques (D.R.E.T.) finance de nombreux travaux sur la cavitation.

Notons également que le Centre National de la Recherche Scientifique (C.N.R.S.) participe à l'effort général par le biais d'une Recherche Coopérative sur Programme (R.C.P.) qui fédère les cinq laboratoires dont les travaux sont évoqués ci-après.

\section{Ecole nationale supérieure de techniques avancées (ENSTA, [58] à [77])}

L'ENSTA est une école d'applications appartenant au Ministère de la Défense et reçoit, à ce titre, des anciens élèves de l'Ecole Polytechnique. Son Laboratoire de mécanique des fluides est commun aux deux écoles. Le Groupe de recherche sur les phénomènes d'interface (GPI) développe au sein de l'ENSTA des travaux dans le domaine de la cavitation en vue de résoudre certains problèmes entrant dans le cadre du génie maritime et du génie industriel. Les principaux thèmes de recherche du groupe sont explicités ci-après.

\section{Erosion de cavitation}

Les études concernant ce problème ont suivi différentes voies. Dans un premier temps, on a procédé à des essais destructifs afin d'observer, au moyen d'un microscope électronique, des empreintes créées par l'implosion d'une bulle sur un matériau préalablement poli et, en multipliant les implosions, la quantité de matière arrachée. Par la suite, on a tenté d'obtenir des estimations énergétiques en effectuant des essais avec des lames de verre calibrées dont la rupture se produisait sous l'effet de l'implosion. Ces différentes techniques ont permis d'interpréter qualitativement certains aspects de phénomène érosif mais il a semblé que d'autres méthodes s'avéraient nécessaires pour aboutir à des estimations quantitatives. C'est dans cet esprit qu'il a été développé une technique d'ellipsométrie rapide ponctuelle destinée à l'étude de contraintes dans un matériau photoélastique soumis à la cavitation.

Les premiers essais ont été effectués en utilisant un laser à une seule longueur d'onde. Il s'est avéré que dans le cas de déformations fortes, l'utilisation d'un laser a deux longueurs d'ondes était nécessaire. Cette installation a été mise en place et des essais de calibration de matériaux photoélastiques ont pu être conduits. Des expériences préliminaires avec des cavités isolées ont aussi été effectuées; on a pu ainsi mesurer des contraintes locales de l'ordre de quelques dizaines de bars à l'intérieur du matériau photoélastique lors du collapse de cavités isolées.

Un programme destiné à la mesure de contraintes à l'intérieur d'un profil soumis à une cavitation par poche courte doit faire suite à l'étude concernant les cavités isolées. Le support financier de l'E.D.F. et de la D.R.E.T. devraient permettre de conduire ces travaux.

\section{Bruit de cavitation}

Le G.P.I. s'est intéressé depuis de nombreuses années au problème du bruit de cavitation et de " signature acoustique " de structures bruyantes. Ces travaux ont été initialement dirigés vers l'interprétation de mesures effectuées au cours du collapse de bulles isolées en fluide indéfini ou en présence d'une paroi solide. Par la suite, on s'est penché sur l'émission sonore associé à des profils en régime cavitant développé avec, comme objectif principal, la détermination des configurations les plus efficaces (niveau sonore) aux basses fréquences (inférieures à $1000 \mathrm{~Hz}$ ).

Grâce à un système d'acquisition et traitement des signaux, il a été possible de conduire une campagne de mesures du bruit de cavitation produits par six profils différents dans un grand nombre de configurations cavitantes. Le niveau d'intensité du bruit a été enregistré pour chaque type de cavitation : par bulles, lames, tourbillons, etc. et la puissance spectrale a été analysée par bandes fines, surtout pour des conditions d'écoulement correspondant aux niveaux sonores les plus élevés. La corrélation entre le bruit émis et le sillage tourbillonnaire a été étudiée pour un profil NACA 16-1512.

\section{Cavitation partielle}

Il s'agit ici d'une étude expérimentale concernant les cavités courtes. Deux aspects du problème reçoivent une attention particulière : d'une part, on s'attache à déterminer par visualisation ultra-rapide le processus de formation, développement et implosion des cavités, d'autre part, on s'intéresse aux dimensions maximales de ces poches.

\section{Cavitation par tourbillons}

Les tourbillons qui se produisent dans les zones de cisaillement intense entre deux écoulements cocurrents, ou bien ceux résultant de la mise en rotation d'ensemble d'une partie de l'écoulement, sont le siège de dépressions pouvant conduire à la mise en cavitation locale du fluide. Exemples de ces situations sont les jets immergés, les sillages derrière des obstacles, les tourbillons marginaux des plans porteurs et des hélices, etc. C'est ce dernier problème qui intéresse tout particulièrement le G.P.I. En effet, l'écoulement tourbillonnaire, dû au caractère portant des profils tridimensionnels en hydrodynamique navale (hélices, safrans, hydroptères, ogives, etc.) provoque, suite à la diminution de pression qui se produit au centre du tourbillon, une cavitation dont la persistance est aussi 
remarquable que celle des tourbillons eux-mêmes. Si la persistance des tourbillons ne présente pas dans les applications marines les mêmes dangers qu'en aéronautique (perte de contrôle des petits avions au passage des tourbillons marginaux de gros porteurs), la cavitation qui y est associée peut conduire d'une part à des dégâts sur des structures en aval, et d'autre part, au rayonnement d'un bruit ayant des caractéristiques propres qui le rendent facilement décelable.

Dans ces conditions, il est intéressant d'envisager des moyens destinés à modifier les caractéristiques cinématiques et dynamiques des tourbillons marginaux de sorte que la naissance et le développement des cavités de vapeur, conduisant à la formation d'un noyau de longueur finie, soient contrôlés. Une telle recherche, outre ses aspects pratiques, développe une meilleure compréhension des problèmes associés et, en particulier, du bruit engendré par les tourbillons marginaux cavitants.

Dans l'étude menée à l'ENSTA, on s'attache à évaluer, au moyen d'expériences simples, les effets introduits par deux techniques: l'injection de solutions de polymères (moyen actif) et l'adjonction de plaque de fermeture ou d'extrêmité (moyen passif).

\section{Institut de mécanique de Grenoble} (IMG, [78] à [97])

Les premières études effectuées à l'I.M.G. ont concerné principalement les écoulements supercavitants et, parmi ceux-ci, les écoulements autour d'ailes à base tronquée, extrados noyé et cavité créée artificiellement par ventilation (études menées en liaison avec le Bassin d'essais des carènes et la S.N.I.A.S. - Marignane), dans le cadre du projet de la Marine nationale de se doter d'hydroptères rapides. Ces études ont permis de définir des formes optimales de profils et de caractériser leurs performances en diverses circonstances de fonctionnement, aussi bien sur le plan de l'analyse théorique que sur le plan expérimental (mesure des efforts subis par une aile, détermination des angles d'incidence exempts de cavitation au bord d'attaque).

Elles ont été menées d'abord en configuration bidimensionnelle, puis sur des ailes tri-dimensionnelles. Elles ont abouti à la proposition de profils mixtes (subcavitants à basse vitesse, supercavitants à grande vitesse). Sur le plan théorique, le schéma fondamental est celui de la théorie classique des sillages en fluide non visqueux, rendu plus maniable cependant soit par la procédure de linéarisation, soit par la méthode des singularités discrétisées.

Parallèlement à ces recherches orientées vers les applications, une autre voie a été explorée dans laquelle on s'intéresse fondamentalement à l'aspect physique des écoulements. Ici, l'expérience joue un rôle prépondérant encore que, dans de nombreux cas, elle soit rendue difficile puisque l'introduction de sondes dans l'écoulement est interdit par l'état quasi instable du liquide proche des conditions de vaporisation. Dans ce cadre, se situent les études suivantes :
- caractères généraux des écoulements à cavités ventilées, étude du mécanisme de pulsation;

- étude de la configuration d'écoulement dite " en demi-cavité " dans laquelle un jet d'eau plan, initialement horizontal, franchit un seuil, se courbe sous l'action de l'influence de la pesanteur et de la différence de pression appliquée sur ses deux frontières libres, puis vient lécher une paroi solide plane peu inclinée sur l'horizontale, déterminant ainsi un espace libre dans lequel l'air est injecté:

- développement de la cavitation dans le sillage de corps épais : cylindres circulaires, coins triangulaires... et modification des caractéristiques du sillage par l'effet de la cavitation;

- étude du détachement d'une cavité largement développée à partir d'une paroi à courbure continue et interaction entre l'état de la couche limite sur la paroi et la cavitation.

Dans les études entreprises plus récemment, qui ont plus spécialement les hélices marines classiques comme champ d'application, on peut noter des travaux concernant la cavitation développée en écoulement instationnaire ou l'érosion de cavitation.

Calcul d'une hélice marine en écoulement instationnaire, avec prise en compte d'une cavitation partiellement développée: Le but de cette étude est de prévoir les efforts instationnaires appliqués sur une hélice marine travaillant dans un champ non uniforme dû au sillage de la carène du navire, avec prise en compte d'une cavitation plus ou moins développée à partir du bord d'attaque des pales.

Erosion de cavitation: On utilise l'érosion de cavitation dans la phase d'incubation (sans arrachement de matériau solide) pour qualifier l'écoulement de liquide quant à sa capacité à produire des structures érosives (bulles, microjets, tourbillons cavitants) à l'arrière d'une poche de cavitation développée. Le comptage du nombre d'impacts produits sur des éprouvettes en écoulement de mercure (I.M.G.) et en écoulement d'eau à échelle 3 pour des vitesses plus grandes (NEYRTEC) permet de tester la validité d'hypothèses de similitude concernant la cinématique des écoulements et leur aggressivité.

Par ailleurs, un modèle a été développé sur l'étude de la réponse d'un matériau solide à un impact de pression.

\section{Institut de mécanique des fluides de Strasbourg (IMFS [98])}

Les travaux effectués sur la cavitation à l'Institut de mécanique des fluides de Strasbourg concernent l'influence des polymères sur les écoulements cavitants sur des obstacles cylindriques et plans à tête cylindrique.

L'adjonction d'un polymère au fluide cavitant se traduit par un retard à l'apparition de la cavitation, par une diminution de l'intensité de la cavitation et par une réduction du bruit de cavitation. L'effet réducteur de la cavitation est attribuée pour une grande partie à la modification globale du champ d'écoulement par le polymère. Ceci présente une certaine analogie avec l'effet réducteur de frottement. Les premiers travaux, à caractère 
technique, avaient pour objectif l'optimisation de la réduction de cavitation sur différents obstacles aussi bien en écoulement homogène qu'en injection pariétale sur l'obstacle. Les recherches se sont ensuite concentrées sur la corrélation étroite entre la réduction de la cavitation et la réduction de traînée dans le cas d'un cylindre circulaire en écoulement non-newtonien homogène.

Les résultats significatifs qui se dégagent de ces recherches sont le retard à l'apparition de la cavitation, la réduction du bruit de cavitation signifiant une diminution de l'intensité de la cavitation et une étroite corrélation entre la réduction de la cavitation et la réduction de traînée. En particulier, le mécanisme de la réduction de cavitation sur un cylindre est essentiéllement une conséquence de la modification par le fluide non-newtonien, d'une part de l'écoulement autour du cylindre et d'autre part de la structure des tourbillons du sillage.

\section{Laboratoire de génie électrique de Paris (LGEP [99] à [116])}

Initialement, le groupe "Cavitation " du LGEP s'était donné comme objectif l'étude des effets électriques consécutifs à la cavitation, notamment par le biais du « microjet " qui suit une bulle mobile en implosion rapide. Celui-ci a deux caractéristiques particulières, sa vitesse (plusieurs centaines de mètres par seconde) et son énergie cinétique de translation conduisant à des pessions d'impact sur une paroi de l'ordre de 10000 bars.

Si l'on admet que le microjet, très rapide, est susceptible de transporter des charges électriques, on pouvait espérer les détecter lors d'un impact sur un capteur. Les essais qui ont été réalisés ont bien fait apparaitre des impulsions de courant de faible durée (inférieure à la micro-seconde) mais la nature de ces charges n'a pas encore été définie. C'est un travail intéressant qui sera repris plus tard.

Parallèlement, on a vérifié que, par son énergie cinétique, la bulle pouvait éroder une surface d'acier inoxydable (par exemple) ce qui se traduit par une variation de différence de potentiel entre cet acier et une électrode de référence. Il se produit vraisemblablement une destruction partielle ou totale de la couche protectrice passivante (oxydes ou autres composés), suivie de sa reconstitution rapide. La loi qui régit ce phénomène est illustrée par une courbe " intensité-potentiel ». Celle-ci est tracée en imposant un courant à travers l'électrode de travail, appelée capteur, et en mesurant son potentiel par rapport à l'électrode de référence.

Ces travaux se sont concrétisés par la conception et la réalisation d'un détecteur de cavitation erosive (DCE) qui doit mesurer l'intensité de l'érosion en un point d'une surface et afficher la vitesse de cette érosion en $\mathrm{mm} / \mathrm{an}$. Il faut noter que l'étalonnage du détecteur est actuellement réalisé avec des érosions produites par meulage ou sablage.

\section{Laboratoire de traitement du signal et ultrasons (LTSU [117] à [119])}

Dans le domaine de la cavitation, le LTSU s'intéresse aux impulsions de pression (ondes de choc) et aux impulsions de lumière produites par les implosions des bulles.

Les impulsions de pression sont détectées par des capteurs piézoélectriques puis enregistrées. Ces impulsions sont ensuite classées suivant leur amplitude pour obtenir un histogramme d'amplitude. Un histogramme résulte de l'analyse d'environ $2 \times 10^{5}$ impulsions. Son intérêt est double. Du point de vue des applications, on sait que l'histogramme d'amplitude est corrélé aux pertes de masse d'un échantillon métallique substitué au capteur. Il faut souligner que l'établissement d'un histogramme nécessite quelques dizaines de seconde tandis que la mesure d'une perte de masse réclame des durées de plusieurs dizaines de minutes voire de plusieurs heures.

L'analyse des histogrammes d'amplitude a également un intérêt pour la modélisation. En effet, cet histogramme présente des zones où les amplitudes des impulsions sont regroupées préférentiellement (pics). Ces pics sont interprétables théoriquement car ils découlent de particularités du mouvement radial. De ce fait, la confrontation des résultats expérimentaux aux prévisions théoriques permet d'avoir une idée sur la valeur de certains paramètres comme, par exemple, la valeur du rayon initial des bulles qui implosent.

D'autres recherches concernent l'étude simultanée des impulsions de pression et des impulsions de lumière consécutives aux implosions des bulles. Là encore, les résultats permettent de préciser la valeur de certains paramètres.

Un autre thème de recherche résulte de la difficulté constatée de la comparaison entre résultats théoriques et expérimentaux dans le cas de l'implosion de bulles isolées. Cette difficulté provient pour une très grande part du fait qu'un grand nombre de bulles implosent en même temps et au même endroit dans une expérience de cavitation. Dans les dispositifs où c'est une bulle unique qui implose (cavitation provoquée par un faisceau laser, bulle produite par la décharge d'une batterie de condensateurs dans des électrodes immergées), le champ de pression n'est pas connu. C'est pourquoi nous avons planifié, depuis longtemps déjà, une série de travaux devant conduire à l'étude de l'implosion d'une bulle unique dans un champ ultrasonore. La première étape consistait en la réalisation d'un générateur de bulles calibrées. Ce générateur est à présent opérationnel. Il combine la production de bulle par soufflage dans un tube capillaire avec le détachement anticipé de ces bulles par un mouvement mécanique. Actuellement, le rayon des plus petites bulles produites est de l'ordre d'une dizaine de microns. La taille de ces bulles est parfaitement reproductible. Dans l'avenir, on utilisera ce générateur pour produire des bulles de rayon initial connu dont on étudiera le mouvement radial quand elles sont soumises à une onde pression. 


\section{Société Neyrtec ([120] à [124])}

La Société Neyrtec, établissement de Grenoble d'Alsthom Atlantique, dispose de sept tunnels de cavitation dont les débits vont de $8 \mathrm{~m}^{3} / \mathrm{s}$ à $3 \mathrm{l} / \mathrm{s}$. La vitesse maximale réalisable et de $60 \mathrm{~m} / \mathrm{s}$ pour un débit de $600 \mathrm{l} / \mathrm{s}$.

Entre 1970 et 1980, l'essentiel des recherches réalisées a consisté à effectuer une étude de l'influence des germes sur la cavitation. Analyse théorique, établissement des règles de similitude à appliquer (concentrations en germes proportionnelles au cube de l'échelle géométrique, en particulier), réalisation des matériels spécifiques nécessaires, telles des méthodes de mesure et de création de germes artificiels, vérification des règles de similitude sur profils et sur hélices de dimensions très différentes.

Les recherches actuellement en cours, relatives au phénomène d'érosion par cavitation, sont effectuées dans un but identique, à savoir la mise en œuvre d'une méthodologie de prévision des risques d'érosion à partir d'essais en modèles réduits, utilisant éventuellement des liquides ou des matériaux différents. Ces recherches sont effectuées en collaboration avec plusieurs autres instituts dont I'ENSTA, I'IMG et le Laboratoire de Matériaux de la DTCN.

Parmi les réalisations de Neyrtec, il faut citer le générateur de tourbillons cavitants dit "veine-tourbillon». Cet appareil avait initialement été réalisé pour montrer que l'érosion de cavitation peut être la conséquence de tourbillons cavitants présentant des noyaux de vapeur (comme ceux produits par la cavitation en lame par exemple). L'appareil comporte une section d'essai dans laquelle on crée un écoulement tourbillonnaire (entrée tangentielle, sortie axiale). Cet écoulement n'est pas cavitant. A un moment donné, on ferme brusquement une vanne amont. Il s'ensuit une onde de pression négative qui rend le tourbillon cavitant. Quand l'onde se réfléchit en aval, elle revient sous la forme d'une onde de pression positive qui provoque l'implosion. Chaque implosion produit un enlèvement de matière (trou). En modifiant les conditions expérimentales, on peut jouer sur l'intensité des implosions.

Autres centres de recherche

D'autres équipes conduisent des recherches sur la cavitation, de manière régulière ou non. C'est ainsi qu'il faut citer le Laboratoire de mécanique des fluides de Poitiers qui dispose d'un tunnel de cavitation à décharge et qui a réalisé diverses expérimentations et analyses théoriques sur des profils tridimensionnels ventilés oscillants ou non. De même, la Société Bertin a étudié certains aspects du bruit de cavitation, en particulier dans le but de développer des sources acoustiques puissantes.

REMERCIEMENTS: Je tiens à remercier chaleureusement les chercheurs et ingénieurs des laboratoires et organismes nationaux qui m'ont apporté une aide efficace pour la mise au point de cet article.

\section{Références}

[1] R.E. APFEL. - Acoustic cavitation, dans Methods of experimental physics, Vol. 19 (Ultrasonics), édité par P. EDMONDS (Academic Press, New York, 1981) chap. 7

[2] E. NIENALTOWSKA. - Phénomènes dynamiques et thermiques pour l'ébullition et la cavitation à l'échelle de la bulle de vapeur. Thèse de $3^{\text {e }}$ cycle (1979).

[3] G.A. ASKAR'YAN, A.M. PROKHOROV, G.F. CHANTURIYA et G.P. SHIPULO. - Sov: Phys. - JETP 17, p. $1463(1963)$

[4] W. LAUTERBORN. - Cavitation and coherent optics, dans Cavitation and inhomogeneities in underwater acoustics, édité par W. LAUTERBORN (Springer Verlag, Berlin 1980) pp. 3-12.

[5] M.G. SIROTYUK. - Experimental investigations of ultrasonic cavitation, dans High-Intensity ultrasonic field, édité par L.D. ROZENBERG (Plenum Press, New-York, 1971) Part. V.

[6] J. FRENKEL. - Kinetic theory of liquids (The Clarendon Press, Oxford, 1946).

[7] D.E. YOUNT. - On the evolution, generation, and regeneration of gas cavitation nuclei J. Acoust. Soc. Am.. 71, n ${ }^{\circ} 6$, pp. 1473-1481, (1982).
[8] M. STRASBERG. - J. Acoust. Soc. Am. 31, pp. 163-176 (1959).

[9] H. MEDWIN. - Ultrasonics, pp. 7-13 (janvier 1977).

[10] F.B. PETERSON, F. DANEL, A. KELLER et Y. LECOFFRE. - Proc. 14th Int. Towing Tank Conference (Ottawa, 1975) pp. 27-52.

[11] W.B. MORGAN. - Proc. 13th Int. Towing Tank Conference (Berlin, Hamburg 1972) 1, p. 657.

[12] J.W. HOYT. - Proc. 14th Int. Towing Tank Conference (Ottawa, 1975), pp. 251-258.

[13] Y. LECOFFRE et J. MARCOZ. - Essais en cavitation à bulles séparées, La Houille Blanche, n 5, pp. 323-329 (1978).

[14] G. CHAHINE. - Etude locale du phénomène de cavitation, analyse des facteurs régissant la dynamique des interfaces, Thèse d'Etat, Université paris VI (1979).

[15] W. BESANT. - A Treatise on hydrodynamics (Cambridge University Press, Cambridge, 1859) p. 198.

[16] Lord RAYLEIGH. - Phil. Mag. 34, $94-98$ (1917).

[17] R.T. KNAPP, J.W. DAILY et F.G. HAMMITT. - Cavitation (Mc Graw Hill, New-York, 1970). 
[18] M.S. PLESSET et A. PROSPERETTI. - Annual Rev. Fluid Mech. 9, pp. 145-185 (1977).

[19] J.M. ROBERTSON. - dans "Cavitation state of knowledge ", A.S.M.E., pp. 1-9 (1969).

[20] S.S. COOK et S.A. PARSONS. - Trans. Inst. Nav. Arch. 61, pp. 233-240, (1919).

[21] C. HERRING. - Columbia Univ. NDRC Report C-4 SR 20-010 (1941).

[22] R. BEECHING. - Trans. Inst. Engr. Shipbuilders Scot. 85, pp. 210-276 (1942).

[23] R.S. SILVER. - Engineering 154, p. 501 (1942).

[24] M. KORNFELD et L. SUVOROV. - J. Appl. Phys. 15, pp. 495-506 (1944).

[25] R.H. COLE. - Underwater explosions (Princeton Univ. Press, 1948, reprinted by Dover Publications New-York).

[26] M.S. PLESET. - Trans. ASME : J. Appl. Mech. 16, pp. 228-231 (1948)

[27] R.T. KNAPP et A. HOLLANDER. - Trans. ASME 70, pp. $419-435$ (1948).

[28] B.E. NOLTINGK et E.A. NEPPIRAS. - Proc. Phys. Soc (London) B.63, pp. 674-685 (1950).

[29] L. TRILLING. - J. Appl. Phys. 23, pp. 14-17 (1952).

[30] F.R. GILMORE, Calif. Inst. of Techn. Hydro. Lab. Report 26-4 (1952)

[31] C.F. NAUDE et A.T. ELLIS. - Trans. ASME 83, Ser. D J. Basic Eng., pp. 648-656 (1961).

[32] H.G. FLYNN. - Physics of acoustic cavitation in liquids, dans Physical acoustics, édité par W.P. Mason (Academic Press, New-York), Vol 1, part. B, pp. 57-172 (1964)

[33] L.W. FLORSCHUETZ et B.T. CHAO. - Trans. ASME 87 Ser. C : J. Heat Transfer, pp. 209-220 (1965)

[34] M.S. PLESSET et R.B. CHAPMAN. - Califormia Inst. of Techn. Div., of Eng. and Appl. Sc. Report 85-48 (1969).

[35] J.M. MITCHELL et F.G. HAMMITT. - Collapse of a spherical bubble in a pressure gradient, ASME Cavitation Forum, (1970).

[36] V.A. AKULICHEV. - Pulsations of cavitation voids, in High intensity ultrasonic fields, édité par L.D. ROZENBERG (Plenum Press, New-York, 1971), Part. IV.

[37] D.Y. HSIEH. - Trans. ASME : J. Basic Eng., 655-665 (1972).

[38] F. NUMACHI. - Trans. ASME: J. Basic Eng. 87, pp. 967-976 (1965)

[39] Specialists Meeting, Cadarache (France) 1976, Int. Atomic Agency, Vienne (Autriche), Rep. IWF GR/9.

[40] M. DEGRóIS et B. BADILIAN. - C.R. Acad. Sc. (Paris) 257, pp. 3333-3335 (1963).

[41] R.M.G. BOUCHER et J. KREUTER. - Chimie et industrie - génie chimique 97, pp. 265-277 (1967).

[42] F.G. HAMMITT et N.R. BHATT. - Univ. Michigan Rep. $N^{n}$ 01357-11-INT (Mod 1) (1972).

[43] A.S. BEBCHUK. - Sov. Phys. Acoust. 3, p. 95 (1957).

[44] A.S. BEBCHUK. - Sov. Phys. Acoust. 3, p. 395 (1957).

[45] M.S. PLESSET. - Trans. ASME, J. Bas. Eng.2, pp. 559-565 (sept. 1972)

[46] A.A. KORTNEV, V.K. MAKAROV et S.G. SUPRUN. Acoustique et ultrasons (Technica Publisher, Kiev) 8, pp. 7-11 (1973).
[47] R. GARCIA, F.G. HAMMITT et R.E. NYSTROM. ASTM Spec. Tech. Publ. 408, pp. 239-279(1967).

[48] R.T. KNAPP, J.W. DAILY et F.G. HAMMITT. - dans Cavitation (Mc Graw-Hill, New-York, 1970) chap. 9.

[49] H. LINDGREN et C.A. JOHNSSON. - Cavitation inception on head forms, ITTC comparative experiments, Pub. of Swedish State Shipbuilding Exper. Tank, n 58 (11 TH ITTC, Tokyo 1966).

[50] J.H.J. VAN DER MEULEN. - A holographic study of the influence of boundary layer and surface characteristics on incipient and developped cavitation on axisymetric bodies, 12th Symposium on Naval Hydrodynamics, Sessions V and Vl (Washington D.C., 1978).

[51] A.P. KELLER et RAMA PRASAD. - Influence of the history of water on cavitation inception, présenté au ASME Cavitation and Polyphase Flow Forum, (Fort Collins, Colorado, 1978).

[52] V.H. ARAKERI et A.J. ACOSTA. - J. Fluids Eng. Trans ASME 95, pp. $519-528$ (1973).

[53] V.H. ARAKERI. - trans. ASME: J. Fluids Eng. 97 pp. 82-86 (1975).

[54] R.E.A. ARNDT et W.K. GEORGE. - Pressure fields and cavitation in turbulent shear flows, présenté au 12 th Symposium on Naval Hydrodynamics (Washington D.C. 1978).

[55] V.K. MAKAROV. - Acoustique et ultrasons (Publication "Technica ", Kiev) 9, pp. 11-14 (1974).

[56] A.A. KORTNEV, V.K. MAKAROV et S.G. SUPRUN. Akust. Zh. 22, pp. 718-723 (1976).

[57] G.L. CHAHINE. - Pressures generated by a bubble cloud collapse, ASME, Cavitation and Multiphase Flow Forum (ST Louis, USA, 1982), pp. 27-31.

[58] G.L. CHAHINE et D.H. et D.H. FRUMAN. - Dilute polymer solution effects on bubble growth and collapse, Physics of fluids 22, 1406 (1979).

[59] G.L. CHAHINE. - Highly distorted bubbles in a Stokes flow, Euromech 99 (Jablona, Pologne, 1979).

[60] G.L. CHAHINE et A. BOVIS. - "Oscillation and collapse of a cavitation bubble in the vicinity of a two-liquid interface ", dans Cavitation and inhomogeneities in underwater acoustics, édité par LAUTERBORN (Springer Verlag, Berlin, 1980), pp. 23-29.

[61] G.L. Chahine, D. COHEN, P. DUCASSE et P. LIGNEUL. - Influence d'un revêtement élastique sur le collapse d'une bulle de cavitation au voisinage d'une paroi solide, $I V^{\prime}$ Rencontre internationale sur la rupture de veine (Cagliari, Italie, 1979).

[62] G.L. CHAHINE et A.K. MORINE. - Collapse d'une bulle de cavitation entre deux parois solides, IV' Rencontre internationale sur la rupture de veine (Cagliari, Italie, 1979).

[63] G.L. CHAHINE et J.S. DARROZES. - Motion of a bubble in a high Réynolds number flow, XIV' Biennal Fluid Dynamics Symposium (Blazejewto, Pologne, 1979).

[64] G.L. CHAHINE, C. COURBIÈRE et P. GARNAUD. Correlation between noise and dynamics of cavitation bubble, 6th Conference on Fluid Machinery (Budapest, Hongrie, 1979).

[65] A. BOVIS. - Asymptotic study of tip vortex cavitation, Cavitation and Polyphase flow Forum (Nouvelle-Orléans, U.S.A., 1980).

[66] E. NIENALTOWSKA. - Cavitation noise emission of cavitating hydrofoils, Cavitation and Multiphase Flow Forum (ASME), (USA, février 1984). 
[67] D.H. FRUMAN. - Tip vortex cavitation inhibition by polymer additives, $C$ and $M F F$ (ASME), (USA, février 1984).

[68] D.H. FRUMAN et N. NIENALTOWSKA. - "Effect of multiple orifices and flow conditions on the cavitation of perforated plates ", C. and MFF (ASME). (USA, février 1984).

[69] D.H. FRUMAN, D. BISMUTH et S. AFLALO. - Effect of polymer solutions on tip vortex cavitation, IUTAM Symposium on the influence of Polymer Additives on Velocity and temperature fields (Essen, R.F.A., 1984).

[70] J.S. DARROZES et G.L. CHAHINE. - Les recherches sur le phénomène de cavitation effectuées à l'ENSTA, Sciences et techniques de l'Armement. Mémorial de l'Artillerie Fransaise, 57 (1983).

[71] G.L. CHAHINE et A.K. MORINE. - The effect of polymer additives on the collapse of a bubble between two solid walls, $C$ and MFF (Nouvelle-Orléans, USA, 1980).

[72] A. BOVIS et G.L. CHAHINE. - Asymptotic study of the non spherical collapse of a bubble near a solid wall, $C$ and MFF (Nouvelle-Orléans, USA, 1980).

[73] A.K. MORINE. - Application de la cinématographie ultrarapide à létude du collapse d'une bulle de cavitation entre deux parois solides, $14^{\circ}$ Conférence Internationale sur la cinématographie ultrarapide, (Moscou, U.R.S.S., 1980).

[74] P. LIGNEUL. - Influence d'un cisaillement de couche limite sur le collapse d'une bulle de cavitation, Euromech 146 (Villard-de-Lans, France, 1981).

[75] P. LIGNEUL. - Dynamique d'un revêtement viscoélastique soumis au collapse d'une bulle de cavitation, $5^{\circ}$ Congrès français de mécanique, (Marseille, France, 1981).

[76] J.S. DARROZES et E. NIENALTOWSKA. - Relative magnitude of phenomena in boiling and cavitating flows, XV Biennal Fluid Dynamics Symposium, (Jachranka, Pologne, 1981),

[77] J.S. DARROZES et P. LIGNEUL. - Drops production by bubble bursting at an air-liquid interface, 2 nd International Colloquium on Drops and Bubbles, (Monterey, California, USA, 1981).

[78] J.M. MICHEL. - Wakes of developed cavities, J. of Ship Research, 21, $\mathrm{n}^{\circ} 4$, pp. $225-238$ (1977).

[79] J. DODU, J.M. MICHEL et A. ROWE. - Les écoulements autour des ailes ventilées, la Houille Blanche, $\mathrm{n}^{\circ}$ 5-6, pp. $449-457$ (1977).

[80] A. ROWE et J.L. KUENY. - Supercavitating hydrofoils with wetted upper sides, $J$. de mécanique 19, $\mathrm{n}^{\circ} 2$ (1980).

[81] A. ROWE et J.L. KUENY. - Recent research on cavity flows about hydrofoils, $13^{\circ}$ Symp. Int. d'Hydrodynamique Navale, O.N.R. (Tokyo, 1980).

[82] J. VERRON. - Ecoulements cavitants autour d'ailes d'envergure finie en présence d'une surface libre, $J$. de Mécanique $18, \mathrm{n}^{\circ} 4$, (1979).

[83] C. PELLONE et A. ROWE. - Etude des caractéristiques d'une aile à base ventilée en théorie non linéaire, 17 colloque d'Aérodynamique Appliquée (Grenoble, France 1980).

[84] C. PELLONE et A. ROWE. - Supercavitating hydrofoils in non-linear theory, $3^{\prime}$ Conf. int. d'hydrodynamique navale numérique O.N.R. - D.G.A. (Paris, 1981).

[85] A. ROWE. - Evaluation of a three-speed hydrofoil with wetted upper side, J. of ship Research 23, $\mathrm{n}^{\circ}$ 1, pp. 55-65 (1979).
[86] J.P. FRANC, J.M. MICHEL et M. LESIEUR. - Structures rotationnelles bi et tridimensionnelles dans un sillage cavitant, C.R.A.S. 295, série II, pp. 773-777 (1982).

[87] J.M. MICHEL, S. BISET et J.P. FRANC. - Cavitation développée sur des parois à courbure continue : Physique du détachement, La Houille Blanche, N ${ }^{\circ} 7-8$, p. 559-570 (1982).

[88] A.R. LAALI et C. PELLONE. - Ecoulements tridimensionnels supercavitants. Traitement numérique en théorie exacte. Expérimentation en Tunnel hydrodynamique, Analyse critique des résultats, La Houille Blanche, $\mathrm{N}^{\circ} 7-8$ (1982).

[89] C. PELLONE et A. ROWE. - Supercavitation hydrofoils in non linear theory, $3^{\circ}$ Conférence internationale d'hydrodynamique navale numérique, O.N.R.-D.G.A. (Paris, 1981).

Les communications [90] à [94] ont été présentées au Colloque Euromech 146 "Flows of liquids past bodies with developed cavities» qui s'est tenu à Villard de Lans (Isère) du 28 au 30 septembre 1981, et qui a été organisé par l'Institut de Mécanique de Grenoble.

[90] J.M. MICHEL. - Some features of ventilated cavities.

[91] J.P. FRANC. - A photographic study of cavitation detachment from a cylinder.

[92] S. BISET. - Etude expérimentale du détachement d'une surface libre à partir d’un profil elliptique: Premiers résultats.

[93] A.R. LAALI. - Air entrainment in ventilated cavities : case of a fully developed cavity formed by a jet of water project over a step up the bottom of a channel.

[94] C. PELlONE. - Non linear calculations of 2-D and 3-D supercavitating hydrofoils : direct problem.

[95] C. PELLONE. - Application de la méthode des singularités au calcul des structures supercavitantes en théorie non linéaire, Thèse, Grenoble (1981).

[96] S. BISET. - Cavitation développée sur une paroi à courbure continue - Etude physique du détachement, Thèse, Grenoble 1982.

[97] J.P. FRANC. - Etudes de cavitation, I - Cascade supercavitante. Calculs inverse et direct. Théorie linéarisée II Sillages cavitants d'obstacles épais. Etude physique. Thèse, Grenoble (1982).

[98] J.M. REINER. - Contribution à l'étude de la cavitation en milieu non newtonien, Thèse Docteur-Ingénieur, Strasbourg (1980).

[99] G. GIMENEZ. - Quelques caractéristiques des impulsions de sonoluminescence, $8^{\circ}$ Symposium international d'acoustique non-linéaire (Paris 1978), paru dans le Journal de Physique, 40, n' 11, p. 289 (1979).

[100] G. GIMENEZ. - Electrical pulses produced by cavitation bubbles, Journal of Physics D: Applied Physics 12, p. 25 (1979).

[101] G. GIMENEZ et A.A. KORTNEV. - Amplitude distribution of pulses produced by shock-waves due to cavitation bubbles, Journal of Acoustical Society of America 67, $\mathrm{N}^{\circ} 4$ (1980).

[102] G. GIMENEZ. - Relation entre la dynamique des bulles de cavitation et les impulsions de pression produites par leurs implosions, Acustica 47, № 2, pp. 142-148, (1981).

[103] G. GIMENEZ et R. SOMMER. - Statistical analysis of hydrodynamic cavitation events, Physics of Fluids, 23, pp. $2140-2141$ (1980)

[104] L. CHINCHOLLE, G. GIMENEZ et F. GOBY. - Electrical effects accompanying cavitation events and detection of erosive cavitation, ASME Polyphase flow in turbomachinery. Winter annual meeting, San Francisco (1978). 
[105] G. GIMENEZ. - Effect of polarization on electrical pulses produced by cavitation bubbles, dans Cavitation and inhomogeneities in underwater acoustics, édité par W. LAUTERBORN (Springer Verlag, Berlin 1980), pp. 101-107.

[106] G. GIMENEZ. - Analysis of pressure pulses produced by cavitation bubbles, Congrès international d'acoustique (Sydney, Australie, 1980).

[107] G. GIMENEZ. - Contribution à l'étude des effets mécanique, lumineux et électrique de la cavitation ultrasonore, Thèse d'Etat, $\mathrm{N}^{\circ} 2046$, Université paris XI, (1978).

[108] F. GOBY. - Etude des courants électriques produits par les bulles de cavitation, Thèse $3^{e}$ cycle, Paris (1978).

[109] R. SOMMER. - Détection des impulsions de pression consécutives à l'action des bulles de cavitation. Thèse de Docteur-Ingénieur, CNAM (1981).

[110] L. CHINCHOLLE, G. KLIMEK, J.J. BONNEFIS et SIMONEAU. - Etalonnage d'un Détecteur de Cavitation Erosive, International symposium AIRH (Amsterdam, 1982).

[111] R. SIMONEAU, J.L. FIHEY et L. CHINCHOLLE. - Effet d'activation anodique de la cavitation érosive, International Symposium AIRH (Amsterdam, 1982).

[112] L. CHINCHOLLE et G. KLIMEK. - Erosion cavitation detector, Sensors (BALE, 1983).

[113] L. CHINCHOLLE. - Répartition du courant à la surface d'une électrode plongée dans un milieu aqueux lorsque cette surface est partiellement abrasée, CRAS PARIS, T. 296, série II-1579, (1983).

[114] L. CHINCHOLLE. - Détecteur d'intensité de cavitation locale, 11 I.C.A., (Paris, 1983).
[115] L. CHINCHOLLE. - Analyse électrochimique de la variation de potentiel consécutive à l'abrasion d'un métal dans un milieu aqueux ", Journées d'Electrochimie 1983, (Paris, 1983).

[116] L. CHINCHOLLE et R. SIMONEAU. - Utilisation industrielle d'un détecteur de cavitation érosive, $X X^{\circ}$ Congrès AIRH, (Moscou, 1983).

[117] G. GIMENEZ. - The simultaneous study of light emissions and shock waves produced by cavitation bubbles, Journal of Acoustical Society of America 71, pp. 839-846, (1982).

[118] G; GIMENEZ, R. SOMMER et C. CACHARD. - Pressure pulses produced by collpasing bubbles, Cavitation and polyphase flow forum (St Louis, U.S.A., 1982).

[119] G. GIMENEZ et C. CACHARD. - Computed and experimental behaviour of cavitating bubbles in presence of a superimposed pressure, Cavitation and polyphase flow forum (Houston, U.S.A., 1983).

[120] P. HENRY, Y. LECOFFRE et P.Y. LARROZE. - Effets d'échelle en cavitation, Symposium AIRH, Tokyo, 1980).

[121] Y. LECOFFRE, B. VALIBOUSE, J. GENEVE et A. BONAZZI. - Some industrial applications of developed cavities, Colloque EUROMECH (Villard de Lans, France, 1981).

[122] Y. LECOFFRE. - Quelques aspects du bruit de cavitation, Symposium GALF-SHF, 1982.

[123] Y. LECOFFRE, J. MARCOZ. - Nuclei and cavitation, Symposium ONR (Ann-Arbor, USA, 1982).

[124] Y. LECOFFRE, J. MARCOZ et B. VALIBOUSE. Generator of cavitation vortex, ASME Symposium Cavitation Erosion in Fluid Systems (Boulder, Colorado, 1981).

\section{Discussion}

Président : M. J. DUPORT

M. Le Président. - Je remercie M. GIMENEZ qui vient de faire un rapport d'une densité très grande; il vous a présenté un panorama extrêmement vaste. Je ne crois pas qu'il soit possible d'avoir à proprement parler une discussion sur cet exposé, pour deux raisons :

- d'une part, M. Gimenez a parcouru un champ extrêmement divers et vaste;

- d'autre part le groupe «Cavitation " en est à la première phase de son travail.

Il faudrait éviter de faire ce soir le travail du groupe « Cavitation " parce qu'on terminerait très tard, et puis qu'est-ce qu'il nous resterait à faire pour la suite ?

Je voudrais tout de même faire quelques observations très générales sur cet exposé.

M. Gimenez a pris soin d'explorer (c'est l'une des tâches du groupe dont il a la responsabilité) au maximum la bibliographie sur non pas le sujet, mais les sujets qui sont à aborder.

Je suggère qu'il serait intéressant de se replonger dans l'ensemble de la documentation résultant des travaux de la Société Hydrotechnique de France car il y a eu sur beaucoup de ces sujets des choses relativement importantes ou très importantes qui se sont écrites peut-être il y a fort longtemps... je pense en particulier aux « figures de cavitation (c'était le terme adopté ici): il y a un travail extrêmement riche de la part des ingénieurs, (et non pas des chercheurs fondamentalistes) sur l'observation de ces figures de cavitation.

Il y a même eu un travail qui reste très important bien qu'assez ancien pour essayer de trouver des corrélations - on était allé assez loin dans ce domaine - entre ce qui est à proprement parler la prévision de l'érosion et les figures observées sur les modèles.
Il y a donc tout un fonds documentaire, en plus de celui que vous êtes en train d'explorer, qui, pour une grande part est celui de la Société hydrotechnique de France.

Il y aura lieu - et je crois que nous en sommes tous bien d'accord - d'approfondir les problèmes qui ont été évoqués, en particulier le problème de la prévision d'érosion de cávitation.

Ce qui est dit dans le texte de M. GIMENEZ concerne la prévision de l'érosion future de cavitation sur des machines déjà existantes qu'on observe avec des "stéthoscopes " particulièrement perfectionnés pour savoir au bout de combien de temps l'érosion va devenir grave. Mais le problème des ingénieurs est un peu plus difficile encore : c'est de prévoir la vitesse d'érosion et les dégâts qui se produiront sur une machine qui n'est pas encore construite et pour laquelle on ne dispose au moment où l'on doit faire cette prévision que de calculs hydrodynamiques et de calculs de projets.

Le recours au modèle réduit pour une telle prévision se heurte aujourd'hui au fait qu'on ne dispose pas de méthodes pratiques de similitude de l'érosion de cavitation, sauf à tomber sur la condition triviale évoquée par M. BONNIN de l'échelle 1, économiquement non applicable quand il s'agit de grandes machines comme c'est le cas en hydroélectricité. Il n'est pas impossible de penser à disposer un jour de méthodes de simulation, sinon de similitude dans ce domaine.

Je pense que par rapport à l'exposé de M. Gimenez également il y aura lieu d'insister davantage sur l'influence de la cavitation plus ou moins développée sur l'écoulement lui-même : ce n'est pas un effet second de l'effet mécanique, c'est l'effet premier auquel les turbiniers ont affaire... Les hydrotechniciens et les turbiniers se sont penchés très tôt sur le problème, car c'est la perte de 
performance des pompes et des turbines qui les inquiétait. Puis, comme ils sont devenus de plus en plus savants et capables, ils se sont autorisés à construire des machines qui, pendant de longues parties de leur exploitation, travaillaient dans des domaines de cavitation plus ou moins développée, et ils ont rencontré les limites imposées par l'érosion. Très souvent en hydraulique, l'art de l'ingénieur est de se situer entre le début de la cavitation, avảnt la chûte de performance, mais en évitant le développement d'une érosion trop rapide.

Etablir ce dialogue entre les chercheurs plus fondamentalistes et les ingénieurs est l'un des buts de ce groupe : il faudrait arriver à ce que les premiers connaissent mieux les problèmes de l'ingénieur (surtout ceux de ces problèmes qui ne sont pas bien résolus) et à ce que les autres, les ingénieurs, approfondissent leur connaissance des phénomènes physiques qui sont présents dans les différentes manifestations de la cavitation.

A ce sujet, je ne crois pas qu'on soit arrivé aujourd'hui, à vraiment être sûr du processus fin qui provoque l'érosion de cavitation. En dehors des approches qu'on pourrait dire " réductionnistes " qu’a évoquées M. GimENEZ, c'est-á-dire celles qui cherchent à expliquer l'érosion par la résorption de la microbulle avec ou sans jet rentrant, - en dehors de cette démarche réductionniste, il en existe dautres qui sont en train de se développer et auxquelles il faudra aussi porter intérêt.

Un mot enfin à propos des applications de la cavitation.

Comme je l'ai dit dans mon introduction, je crois qu'il faut attacher beaucoup d'intérêt aux suggestions de M. GIMENEZ à ce propos...

Aujourd'hui - soyons francs - il y a très peu d'industries dont l'activité est fondée sur les applications de la cavitation. Par contre il y a beaucoup d'industries florissantes, en France en particulier, qui sont de celles qui cherchent plutôt à reculer les limites de la cavitation. Eh bien! je crois que des contacts plus approfondis entre ingénieurs et scientifiques pourraient être générateurs d'idées nouvelles permettant de faire des innovations et, pourquoi pas, de voir surgir des petites entreprises ou des entreprises moyennes innovatrices qui justement cultiveraient les applications positives de la cavitation.

Je crois avoir ainsi présenté les principales remarques que je souhaitais faire et l'on peut ouvrir une brève discussion.

M. SAMADEN. - Est-ce qu'il y a moyen de mesurer la densité des germes par unité de volume ?... Ma question concerne aussi le modèle à plusieurs bulles pour modéliser la cavitation.

M. GIMENEZ - C'est un travail de groupe.

M. LECOFFRE. - II y a un certain nombre dappareils qui permettent de mesurer les germes. Leur utilisation dépend des besoins propres des utilisateurs. Des synthèses ont été faites dans les compte-rendus de l'ITTC.

Parmi les méthodes qui existent, il y a des méthodes optiques diffraction locale, diffraction à l'infini, holographie; il y a des méthodes basées sur des phénomènes électriques (coulter counter); il y a aussi un certain nombre de méthodes appelées "directes".
Les premières méthodes que j’ai citées donnent une estimation de la taille des particules dans l'eau; mais il y a des méthodes dites "directes " : on fait caviter les germes contenus dans l'eau à des niveaux de pression négative (ou de tension) variable. Dans ces conditions on détermine le nombre de germes qui, sous un niveau de pression donné, donnent naissance à des bulles de cavitation. Cela permet de se faire, avec un certain nombre de limitations tout de même, une bonne idée de la "qualité de l'eau ".

En ce qui concerne votre question sur les modèles à plusieurs bulles un certain nombre de modèles théoriques ont été développés. De tels modèles ont été faits par M. CHAHINE. Nousmêmes avons développé quelques modèles de grossissement de bulles qui permettent d'estimer les pertes de performance qu'auront les profils hydrodynamiques, dans certains cas particuliers.

M. GIMENEZ. - Vous avez la référence du papier relatif aux germes dans mon exposé... c'est ce à quoi faisait allusion $\mathrm{M}$ LECOFFRE.

M. Le Président. - Faire le point, ce n'est pas seulement faire le point des connaissances d'un côté, et le point de l'état des solutions des problèmes de l'autre. C'est aussi identifier les sujets de recherche: mais c'est également - et c'est probablement encore plus important - faire le point de l'avancée des méthodes.

S'agissant des méthodes de mesure des germes, nous étions il y a pas mal d'années intéressés à des méthodes optiques qui n'étaient pas évidemment opérationnelles pour le sodium liquide... qui n'est pas, je pense étranger à vos préoccupations... Faire le point sur les méthodes de mesure des germes sera l'une des tâches du groupe et je rappelle à ce propos que l'un de nos objectifs, c'est qu'un certain nombre d'entre vous non présents aujourd'hui dans le groupe viennent le rejoindre pour y apporter leur contribution.

M. BONNIN. - Il y a un point que je voudrais relever; il a fait l'objet d'un aparté entre M. Mondin et moi-même : c'est que la définition que vous avez donnée de la cavitation s’applique exactement à l'ébullition.

Ce n'est pas une critique contre votre exposé, c'est une critique contre les phénomènes. Nous-mêmes, plus nous avons fait de cavitation, moins nous avons été capables de définir les phénomènes; et plus nous avons fait de la cavitation, plus nous avons été proches de l'ébullition.

M. MICHEL - M. Gimenez a privilégié l'approche bulle unique. Mais il existe aussi la "cavité " développée, accrochée à des parois solides; et parfois les gens peuvent être en présence des deux phénomènes. Il y a eu de nombreux essais en vue de la modélisation de telles poches. Des problèmes subsistent notamment en écoulement tridimensionnel d'une part et d'autre part en cavitation instationnaire. Il y a des représentations non linéaires qui se révèlent actuellement assez performantes.

M. Le Président. - Je vous remercie non seulement de votre exposé M. GIMENEZ, mais aussi d'avoir pris la charge de ce groupe. et je vous souhaite bonne continuation.

\section{Les activités du groupe de travail Cavitation}

La constitution du groupe de travail "cavitation" a été décidée en mai 1982 par le Bureau du Comité Technique de la SHF. Depuis cette date, le groupe s'est réuni à quatre reprises.

Trois thèmes de travail ont été définis :

- Cavitation développée (incluant la question des performances des machines et les problèmes posés par les profils mais excluant l'érosion);

- Bruit de cavitation (incluant le problème des vibrations);

- Erosion de cavitation (incluant la question du comportement des matériaux vis à vis de la cavitation).

Les activités du groupe comprennent :

- des échanges d'informations (sources de documentation, principaux centres de recherches...);

- des exposés généraux, comme par exemple, l'aspect métallurgique de l'érosion par cavitation ou la modélisation des poches développées;

- des exposés sur les recherches, les études ou les essais conduits par les participants:

- des exposés sur l'approche industrielle des problèmes de cavitation:

- des débats sur des questions relevant des thèmes précédemment mentionnés.

En outre, le groupe espère, à terme, pouvoir établir un « Etat de' connaissances " sur la cavitation. 


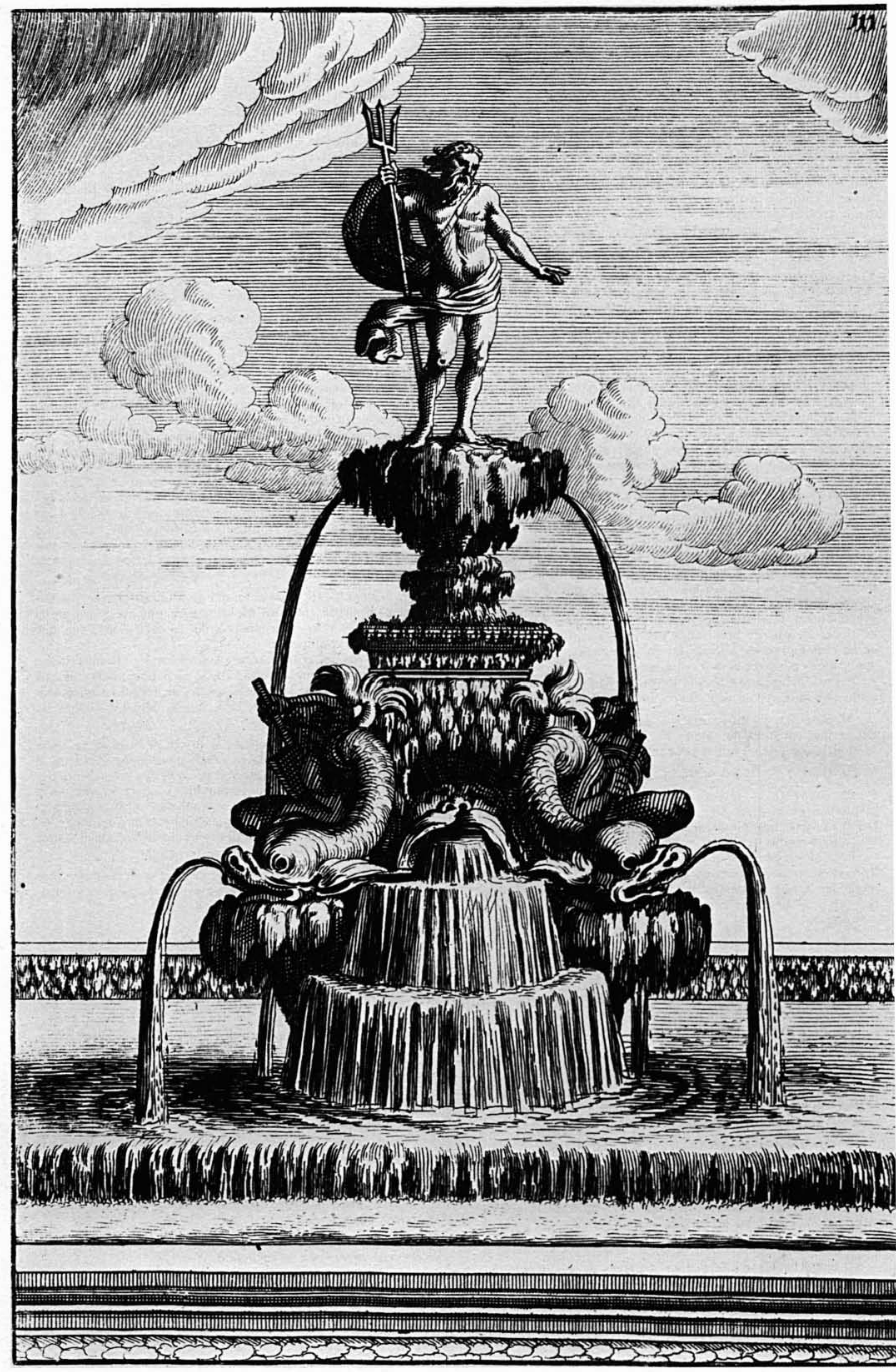

